\title{
Accumulation of charges in polycarbonate due to positron irradiation
}

\author{
D. Cangialosi ${ }^{\mathrm{a}, *}$, H. Schut ${ }^{\mathrm{b}}$, M. Wübbenhorst ${ }^{\mathrm{a}}$, J. van Turnhout ${ }^{\mathrm{a}}$, A. van Veen ${ }^{\mathrm{b}}$ \\ ${ }^{a}$ Interfaculty Reactor Institute, Delft University of Technology, Mekelweg 15, $2629 \mathrm{JB}$ Delft, The Netherlands \\ ${ }^{\mathrm{b}}$ Department of Polymer Materials and Engineering, Delft University of Technology, Julianalaan 136, 2628 BL Delft, The Netherlands
}

\begin{abstract}
Positron annihilation lifetime spectroscopy is a powerful technique for the study of free volume in polymers. The lifetime of ortho-positronium (o-Ps), a bound state of an electron and a positron, can be used to assess the cavity size, while the intensity can be used to characterize the number of cavities. In the past, results have been published, which suffer from artifacts, whereby the drop in $o$-Ps intensity is not related to a decrease in the number of cavities. One of the possible artifacts is the build-up of an internal charge during long-term exposure to positron irradiation, which affects the $o$-Ps intensity. In this study, we focus on the charging created in polycarbonate by positron radiation, which we investigated by the laser intensity modulation method and by charge-decay experiments, both isothermally and with thermal stimulated discharge. From these measurements we conclude that: (1) the $o$-Ps intensity decrease during prolonged positron irradiation is due to the accumulation of a space charge; (2) in reverse, changes in the $o$-Ps intensity may be used to probe electric fields in dielectrics.
\end{abstract}

(C) 2003 Elsevier Science Ltd. All rights reserved.

Keywords: Positron irradiation; o-Ps intensity; Charging

\section{Introduction}

For the characterization of amorphous polymers, the determination of the free volume plays an important role. Among the techniques which yield information about the free volume, positron annihilation lifetime spectroscopy (PALS) is the most direct, since it allows the detection of free volume holes on an atomic scale. When positrons are injected into a polymer, they can form positronium (Ps) in the two states: ortho- and parapositronium (o-Ps and $p-\mathrm{Ps})$, the triplet and the singlet state of the system positron-electron (Mogensen, 1995). The $p$-Ps lifetime is very short (about $125 \mathrm{ps}$ in vacuum). Annihilation occurs with the emission of two gamma photons at $0.511 \mathrm{MeV}$. In contrast, $o$-Ps is a long living system with a lifetime of about $142 \mathrm{~ns}$ in vacuum. Its annihilation takes place with the emission of three

\footnotetext{
*Corresponding author. Tel.: + 31-15-2784535; fax: +31-152786422.

E-mail address: d.cangialosi@tnw.tudelft.nl (D. Cangialosi).
}

gamma photons. Interestingly, in condensed matter such as polymers, $o$-Ps is easily trapped in the potential well of a free volume cavity. Annihilation then occurs after localization of the positron with an electron of opposite spin on the wall of the cavity. This process, known as pick-off reaction, strongly reduces the lifetime of $o$-Ps, $\tau_{o-\mathrm{Ps}}$, to values between 1 and $5 \mathrm{~ns}$. Since $\tau_{o-\mathrm{Ps}}$ is inversely proportional to the square of the overlap of the positron component of the Ps wave function with the cavity wall electron wave function, it is related to the size of the cavity itself. A semi-empirical equation (Eldrup et al., 1981) was obtained that correlates $\tau_{o \text {-Ps }}$ with the radius of the cavity $R$, assuming a spherical potential well of radius $R_{0}$ with an electron layer of thickness $R-R_{0}=1.656 \AA$.

$\tau_{o-\mathrm{Ps}}=\frac{1}{2}\left[1-\frac{R}{R+\Delta R}+\frac{1}{2 \pi} \sin \left(\frac{2 \pi R}{R+\Delta R}\right)\right]^{-1}[\mathrm{~ns}]$.

The probability of $o$-Ps formation is called the intensity $o$-Ps, $I_{o-\mathrm{Ps}}$, which is proportional to the number of cavities in the system (Kobayashi et al., 1989). In this 
manner, the total free volume can be evaluated from the following equation:

$v \mathrm{f} \propto \frac{4}{3} \pi R^{3} I_{o \text {-Ps }}$

Until now, a few PALS studies have been reported which attempt to relate free volume variations to the structural relaxation of polycarbonate (PC) (Hill and Agrawal, 1990; Sandreczki et al., 1996; Davies and Pethrick, 1998). In these papers, a drop in $I_{o-\mathrm{Ps}}$ is attributed to the reduction of the concentration of free volume elements. Unfortunately, prolonged positron irradiation was proven to be responsible for most of the $I_{o \text {-Ps }}$ drop as was demonstrated by other authors ( $\mathrm{Li}$ and Boyce, 1993; Qi et al., 2001; Chen et al., 2001). All of them observed a reduction of $I_{o-\mathrm{Ps}}$ that cannot be ascribed to any structural relaxation, since $I_{o \text {-Ps }}$ returns to its original value if the sample under investigation is replaced by another one with the same thermo-mechanical history, but free of irradiation effects. According to these authors, the main reason for this interference of $o$ Ps formation is the build-up of an internal electric field, due to the lack of charge dissipation in the insulating polymer. However, to the best knowledge of the authors, no papers have appeared yet, which give direct evidence for electrical fields induced in the sample after prolonged positron irradiation. The aim of this work is to characterize the charge distribution in PC samples after exposure to a positron source. The techniques used are the thermal stimulated discharge (TSD), to determine the surface potential and its post-irradiation decay, and a thermal wave technique (laser intensity modulation method-LIMM) to determine the charge profile across the thickness of the sample.

\section{Experimental}

PC from General Electric (Lexan 161) was used in all the experiments. The polymer was first dried at about $120^{\circ} \mathrm{C}$ in vacuum and then compression molded to sheets of 1 and $4 \mathrm{~mm}$ in thickness. Specimens of about $1 \mathrm{~mm}^{2}$ area were taken from each sheet. Samples were positron irradiated as obtained or after metallization with gold on one side. It is worthwhile noting that the metallized samples were irradiated on their non-metallized side.

PALS measurements were performed by exposing the samples to the radioactive isotope ${ }^{22} \mathrm{NaCl}$ with an activity of $10 \mu \mathrm{C}$. The irradiation time was varied between 2 and 15 days. A fast-fast coincidence circuit of PAL spectrometer with a lifetime resolution of $240 \mathrm{ps}$ monitored by using ${ }^{60} \mathrm{Co}$ source was used to record all PAL spectra. The counting rate was about $70 \mathrm{cps}$. Each spectrum was collected to a total count of $2 \times 10^{6}$. This resulted in an acquisition time of $9 \mathrm{~h}$ for each spectrum. Such an exposure time was proven to result in negligible radiation effects as illustrated by Fig. 2a. All spectra were analysed using POSITRONFIT (Kirkegaard et al., 1981), which describes the spectrum as a convolution of the instrument resolution function and a finite number of negative exponentials plus the background:

$y(t)=R(t) \otimes\left(N \sum_{i=1}^{n} I_{i} \lambda_{i} \exp \left(-\lambda_{i} t\right)+B\right)$.

Here $R(t)$ is the resolution function, $\lambda_{i}$ the inverse of the annihilation times (annihilation rates), and $B$ the background. All spectra were decomposed in three lifetime components: a short one related to $p$-Ps annihilation, an intermediate one related to free positron annihilation, and a long component related to $o$-Ps annihilation.

After positron irradiation, the isothermally or thermally stimulated decay of the surface potential was monitored using the capacitive probe technique shown in Fig. 1.

The surface voltage yields the total charge density within the sample. To determine the spatial distribution of the charge we applied the LIMM, a thermal wave technique. The experimental set-up used is described in (Wübbenhorst and van Turnhout, 1994; Quintel et al., 1998). LIMM is based on pyroelectricity, a property of dielectric materials that show a temperature-dependent polarization $P$; they therefore generate a current as a result of a temperature change $\Delta T(t)$. The pyroelectric current density of a charged sample covered with two electrodes is given by: $j(t)=p \mathrm{~d} T / \mathrm{d} t$, where $p$ denotes the pyroelectric constant: $p=\mathrm{d} P / \mathrm{d} T$. The latter is proportional to the electric field, and so the charge profile can be calculated from the LIMM spectra.

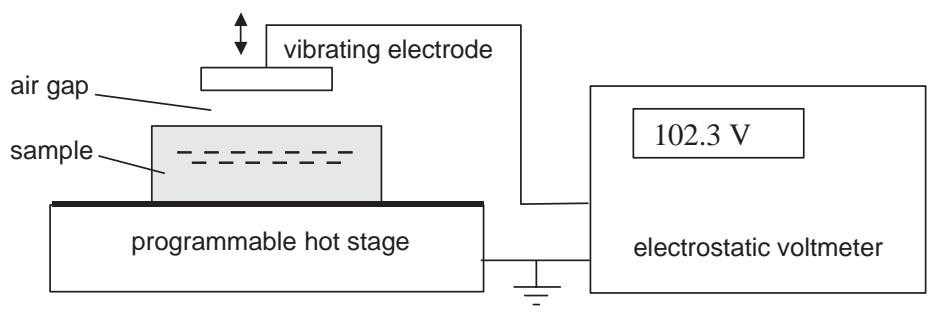

Fig. 1. Schematic representation of the capacitive probe method. 


\section{Results and discussion}

Figs. 2 and 3 show the course of $I_{o \text {-Ps }}$ during irradiation and the subsequent charge decay measured with the capacitive probe. Fig. 2 applies to a nonmetallized 4-mm thick sample, while Fig. 3 relates a metallized 4-mm sample. The non-metallized sample shows a marked decrease in $I_{o-\mathrm{Ps}}$ during positron irradiation, which translates to a gradual charge buildup (voltage measured after radiation is $2000 \mathrm{~V}$ ). The charge accumulated is stable at room temperature and is released only near the glass transition temperature $\left(T_{\mathrm{g}}\right)$. In contrast, the metallized sample does not show any
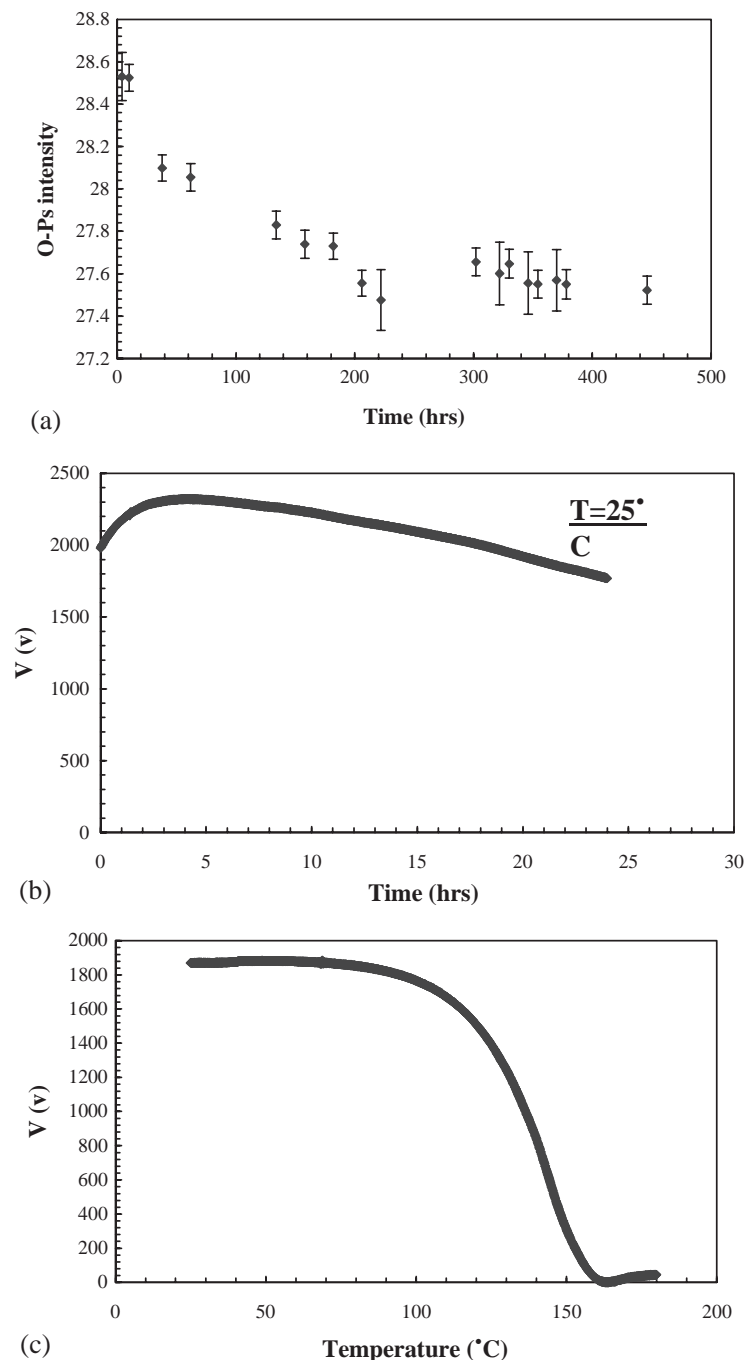

Fig. 2. Response of the $4 \mathrm{~mm}$ non-metallized sample to positron irradiation and its charge decay: (a) o-Ps intensity decay vs. irradiation time; (b) room-temperature charge evolution; and (c) thermally stimulated discharging. The amount of charge is measured in terms of electrostatic potential.

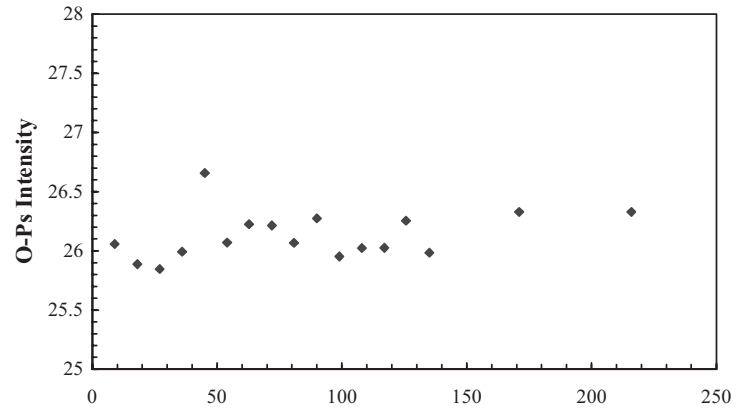

(a)

Time (hrs)

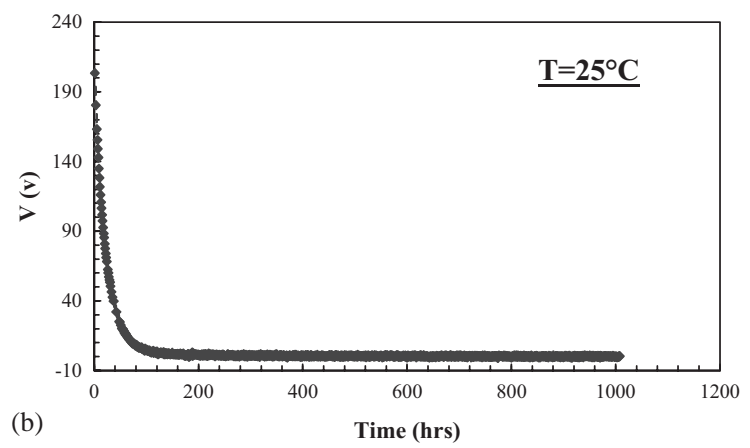

Fig. 3. Response of $4 \mathrm{~mm}$ metallized sample to positron irradiation and its charge decay: (a) $o$-Ps intensity behaviour; (b) discharging at room temperature. The amount of charge is measured in terms of electrostatic potential.

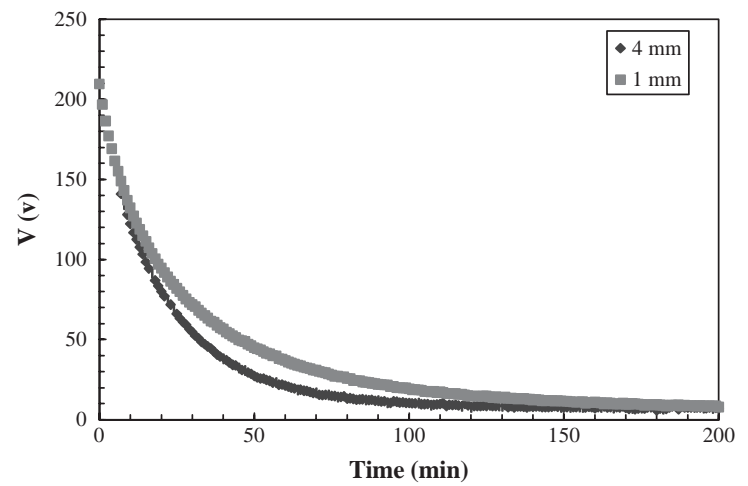

Fig. 4. Comparison between discharge rate of 1 and $4 \mathrm{~mm}$ metallized samples.

effect on $I_{o \text {-Ps }}$, since it remains practically constant during the irradiation. The absence of an effect on $I_{o \text {-Ps }}$ is in line with the charge that shows up at room temperature. Two striking features can be seen in Fig. 3b: (i) the initial value of the potential is low, about one order of magnitude less than that of the nonmetallized sample; (ii) the charge rapidly decays to zero in a couple of hours, even at room temperature. Fig. 4 


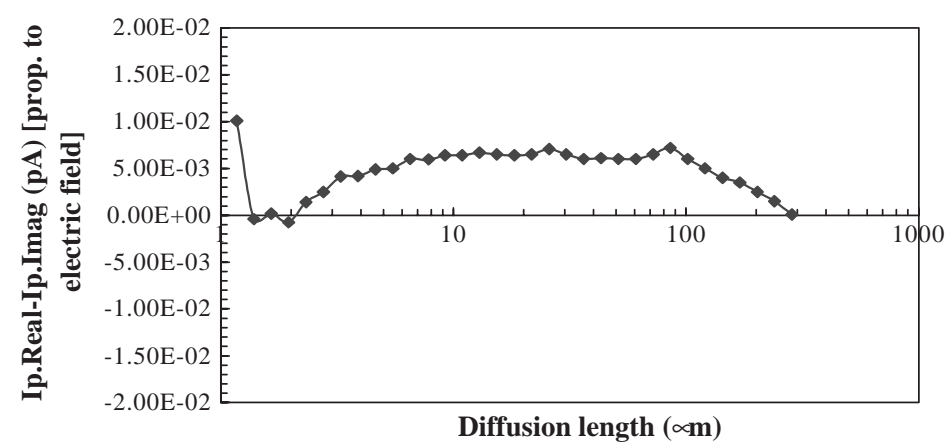

Fig. 5. LIMM plot for the non-metallized sample, which describes the charge profile after positron irradiation.

suggests that there is a slight difference between the $1 \mathrm{~mm}$ - and the $4 \mathrm{~mm}$-thick metallized sample. The thinner sample has a higher initial value and a slightly different decay profile. However, this difference becomes negligible if compared with the non-metallized sample, which shows no charge release at all at room temperature.

Evidently, the presence of a metal layer strongly hinders the storage of charge and provokes a fast decay of the small amount of charge retained. By contrast, charge is much more efficiently trapped in the nonmetallized sample. The charges created in the metallized samples are rapidly dissipated by ohmic conduction due to the increase in the bulk conductivity of PC by the irradiation with the gamma photons generated both from the positron annihilation and from the ${ }^{22} \mathrm{Na}$ radioactive decay.

The charge distribution revealed by LIMM also looks interesting. This is shown in Fig. 5, where the difference between the real and imaginary part of the pyroelectric current is plotted across the thickness of the insulated sample, in which the charge is retained after irradiation. The results demonstrate a variation in the polarization, which corresponds to the presence of a space charge accumulated between 0.1 and $0.3 \mathrm{~mm}$ from the surface.

\section{Conclusions}

We proved the build-up of an internal electric field in PC samples due to prolonged positron irradiation. Its effect on the positron response was determined through the analysis of the probability of Ps formation $\left(I_{o-\mathrm{Ps}}\right)$, while the corresponding charge pattern was analysed by surface potential decay and thermal wave measure- ments. The results show a clear influence of the sample layout on the development of the trapped charge both during and after irradiation. Particularly, the presence of a metal layer on the back of the irradiated sample induces a rapid neutralization of the created charge independent of the thickness of the sample.

\section{References}

Chen, Z.Q., Suzuki, T., Kondo, K., Uedono, A., Ito, Y., 2001. Jpn. J. Appl. Phys. 40 (8), 5036-5040.

Davies, W.J., Pethrick, R.A., 1998. Eur. Polym. J. 34 (12), 1747-1754.

Eldrup, M., Lightbody, D., Sherwood, J.N., 1981. Chem. Phys. 63 (51), 51-58.

Hill, A.J., Agrawal, C.M., 1990. J. Mater. Sci. 25 (12), 5036-5042.

Kirkegaard, P., Eldrup, M., Mogensen, O.E., Pedersen, N.J., 1981. Comput. Phys. Commun. 23, 307-335.

Kobayashi, Y., Zheng, W., Meyer, E.F., McGervey, J., Jamieson, A., Simha, R., 1989. Macromolecules 22 (5), 2302-2306.

Li, X.S., Boyce, M.C., 1993. J. Polym. Sci., Polym. Phys. 31 (7), 869-873.

Mogensen, O.E., 1995. Positron Annihilation in Chemistry. Springer, Berlin.

Qi, C.Z., Ma, D., Hu, Y.H., Yan, F.Y., Gao, H., Yang, Z., Zhou, X.X., Wang, T.M., 2001. J. Polym. Sci. Polym. Phys. 39 (3), 332-336.

Quintel, A., Hulliger, J., Wübbenhorst, M., 1998. J. Phys. Chem. 102 (22), 4277-4283.

Sandreczki, T.C., Hong, X., Jean, Y.C., 1996. Macromolecules 29 (11), 4015-4018.

Wübbenhorst, M., van Turnhout, J., 1994. Proceedings of the Eighth International Symposium on Electrets, Paris, pp. 182-187. 